\title{
Ant Colony Optimization (ACO) based Routing Protocols for Wireless Sensor Networks (WSN): A Survey
}

\author{
Anand Nayyar \\ Ph. D (Computer Science) Research Scholar \\ Desh Bhagat University, Mandi Gobindgarh
}

\author{
Rajeshwar Singh \\ Director, Doaba Group of Colleges, Nawanshahr
}

\begin{abstract}
Wireless Sensor Networks have several issues and challenges with regard to Energy Efficiency, Limited Computational capability, Routing Overhead, Packet Delivery and many more. Designing Energy Efficient Routing Protocol has always been a limiting factor cum issue for Wireless Sensor Networks. Varied routing protocols being proposed till date for overcoming these issues based on Swarm Intelligence. Swarm Intelligence is concerned with study of combined behavior of systems designed by varied components for coordinating among themselves via decentralized controls and self-organization. Algorithms based on Swarm Intelligence, nature based intelligence are highly robust, adaptive and scalable. This paper presents comprehensive survey of Ant Colony Optimization based routing protocols for Wireless Sensor Networks to give better platform for researchers to work on various shortcomings of protocols developed till date to develop efficient routing protocol for WSN in near future.
\end{abstract}

Keywords-Wireless Sensor Networks; Routing; Routing Protocols; Swarm Intelligence (SI); Ant Based Routing; Ant Colony Optimization

\section{INTRODUCTION}

In 21st Century, Wireless Sensor Networks (WSN) is regarded as one of the fastest evolving technologies. Wireless Sensor Network is enabled by recent sophisticated advancements in MEMS and Wireless communication technologies. A Wireless Sensor Network infrastructure is composed of nodes working autonomously with sensing, computation and networking elements which enables end user to measure, monitor and act to various events or phenomenon in different environments [1,2]. Wireless Sensor Networks features tiny, compact-size, and inexpensive computational wireless transmitting nodes with limited energy scattered over the area with diverse parameters like store and forward the data to central location for further processing. WSN nodes have the ability to operate in any sort of environment and network with other nodes to carry out data transmission tasks. WSNs can be easily deployed in various applications including civil, military, environmental monitoring, surveillance, health care applications, industrial production, transportation, space technology and more.

The implementation of Wireless Sensor nodes is done in ad-hoc manner without any sort of appropriate planning or research. Once deployed in real-world, the sensor network with other nodes autonomously transmit data back to sink node using different routing protocols. WSN nodes are battery powered, so limited energy is always a barrier, as it becomes difficult to change or recharge the batteries of sensor nodes in live operational environments [3].

Sensor nodes have limited transmission range and both data and control packets have to route from node to node back to sink node only via multi-hop fashion way [39]. Despite of varied objectives of sensor network applications, the primary function of WSN node is to collect specific data from the environment, process and transmit the data back to sink node via radio transmitter. In order to facilitate effective transmission of nodes among each other, the routing protocols should be highly efficient. A large number of challenges and issues in terms of theory and practical are taken into account to make the routing protocols efficient like maximization of network life-time, efficient route-discovery as nodes are autonomous which means that protocols must be selforganizing and protocols must handle random and complex environments and all sorts of radio interference to easily discover and maintain efficient Multihop routing paths [4].

Traditional or Classical routing protocols of Wireless Sensor Networks (WSNs) are not energy efficient and scalable to face varied challenges in dense and complex environments like military, production, medical and many other scenarios. So, there is an emergent need of new routing protocol based on Swarm Intelligence based technique for performing efficient routing and maintaining energy efficiency among nodes during data transmission. Till today, enormous types of routing protocols have been proposed for WSN based on varied social insects like Ants, Birds, Cats, Dog, Bats, Elephants on basis of their real world working mechanisms and optimizations. The goal of this research paper is to review the most prominent routing protocols based on Ant Colony Optimization (ACO) for WSN.

Swarm Intelligence [5-9] is a novel area considered for development of various optimizations in diverse areas. Currently, SI is regarded as important and foremost choice for every researcher across nook and corner in the world to develop efficient routing protocols for WSN/MANETS/VANETS/FANETS and many more areas.

Swarm Intelligence is a group of homogenous individual agents, having capability of self-organization, interaction among themselves and with environment. 
Swarm Intelligence is originally defined as "Any Attempt to design algorithms or distributed problem-solving devices inspired by collective behavior of social insects and other animal societies".

Swarm Intelligence was first conceptualized by G.Beni, Hackwood and J.Wang in 1989. Swarm Intelligence is primarily focused on study of integrated behavior of social insects as well as other animal societies using decentralized controls and self-organization. Swarm Intelligence enables tremendous increase in performance, robustness, scalability and efficiency by providing solution to complex problems. SI being an important concept in field of Artificial Intelligence and Computer Science focusses on development of various algorithms to deploy many simple agents with no rule and leading to global behavior using Ants, Birds, Honey bees etc. The interactions between the individual swarms can be direct or indirect. Direct interactions can be done via mode of audio or Video. Example: Birds interact among each other via making specific sounds and Bees interact with their communities using Waggle Dance. Indirect interactions mean interaction via environment i.e. One Swam Agent change the environment and other swarm responds to the changing Environment. Example: Ant Colony Optimization (ACO).

Swarm Intelligence based Techniques have widened scope in area of WSN. The most widely used techniques are Ant Colony Optimization and Bee Colony Optimization as the routing protocols based on ACO or Bee Colony are highly efficient in varied parameters like energy, robustness, scalability thus perform better in complex transmission environments.

\section{A. Organization of Paper}

The rest of the paper is organized as follows. Section 2 summarizes various Design challenges cum issues faced in designing routing protocols for WSNs. Section 3 briefly overviews the concept of Ant Colony OptimizationMetaheuristic, Algorithm and Implementation with Wireless Sensor Network. Section 4, presents detailed review of various selected routing protocols based on Ant Colony Optimization for Wireless Sensor Networks. Finally, Section 5 concludes the paper with directions for future research.

\section{CHALLENGES DURING DESIGN FOR ROUTING PROTOCOLS OF WIRELESS SENSOR NETWORKS}

A tremendous research has been carried out by researchers to overcome various challenges in WSN for successful real world operations.

WSNs being composed of tiny nodes having limited memory, low-processor, less-energy and small-bandwidth capabilities in-turn face strict constraints while designing routing protocols. WSNs transmit voluminous amounts of data back to sink node which makes use of energy, bandwidth and computing power. As sensor nodes operate in dynamic environment, large number of challenges are also faced in terms of architecture.

So, the utmost requirement is to design energy efficient routing protocols to overcome various additional challenges like: Power, Mobility, Connectivity, QoS, Data Aggregation,
Deployment, Security, Cost, Congestion, Latency, Localization and many more.

The following are some of the issues which are to be taken into serious consideration by researchers to design Efficient Routing Protocol for WSN [2, 10, 11, 12, 13]:

- Less Computation and Limited Memory Requirements: Sensor nodes comprising Wireless Sensor Networks are equipped with Low-power CPU and tiny Memory in $\mathrm{KBs}$ like ATMega Processors containing $16 \mathrm{~KB}$ to $128 \mathrm{~KB}$ memory. So, Routing protocols should be designed consuming less CPU-power and execution should be feasible in limited amounts of memory.

- Deployment: Sensor nodes being deployed in random manner without any planning or research and are application dependent which ultimately affects the overall performance of routing protocol. So, in order to deal with ad-hoc environment, the routing protocol should be competent enough to self-organize the nodes and establish the paths among each other for data transmission and energy consumption.

- Energy Efficiency: The primary challenge for every routing protocol deployed in sensor nodes is Energy Efficiency. Sensor nodes exhaust the energy while performing varied tasks like Sensing, Computation and Transmission. It becomes utmost important that routing protocol should (1): Discover effective paths among nodes for transmission among each other and sending back the data to sink node. (2): Allocate the forwarding of data packets across multiple paths i.e. Effective multi-path routing should be integrated. (3): Activation of only those nodes used in transmission and keeping non-transmitting nodes in sleep mode.

- Scalability: As sensor network comprise of hundreds to thousands of nodes deployed for sensing the environment, mark utmost requirement i.e. routing protocol should be scalable enough to handle and respond to diversified events.

- Fault Tolerance: Lots of issues can occur in real world to sensor nodes in terms of power failure, physical damage, radio interference which overall affects the entire WSN network performance. Routing protocols should be fully compliant and compatible to handle these issues and should generate new routes among nodes to sink nodes in case of any node failure in between so that transmission can work without any sort of hic-cups.

- Latency/End-To-End Delay: Latency, in simple terms, is regarded as the time taken by a data packet to reach from node to sink node. Latency is measured in: OneWay: The time from source to sink, Round-Trip: The one-way latency from source to sink and from sink back to the source. Apart from this, latency can also be caused by Multi-Hop relays and data aggregation. So, routing protocol should be fully efficient and should take less time in transmission of data from nodes to sink nodes which also reduces the problem of 
congestion and packet failure in overall network operation.

- Quality of Service (QoS): Another challenge in effective design of routing protocol is Quality of Service (QoS). While designing routing protocol, QoS parameters like Jitter, Bandwidth, Delay and Reliability should be considered so that various aspects like data reliability, energy efficiency, collaborative processing can be maintained in WSN network.

- Data Gathering and Aggregation: Data aggregation and gathering can be event-driven, query-driven, continuous or hybrid combination. Data gathering methods occupy significant position in WSN routing, as after getting data, the node has to transmit the data back to sink node.

In lieu of various issues discussed above, researchers have proposed different protocols for WSN with regard to routing optimization as routing in WSN, till date, is surrounded by lots of challenges and constraints. Proposed routing protocols consider various sensor node characteristics with regard to application and architecture.

\section{NATURE BASED ROUTING-ANT COLONY OPTIMIZATION-OVERVIEW AND ALGORITHM}

Mostly the routing protocols being proposed and developed by researchers for Wireless Sensor Networks based on SI are based on Ants \& Bee Colonies. The foraging behavior of insect societies is regarded as the major source of backbone to design highly energy efficient and sophisticated routing protocols for WSN.

Ants, during process of foraging, collectively explore the environment to find traces of food sources, and once food source is located, ant's setup paths between the nest and food sources to effectively transport the food back to nest. Therefore, the collective foraging behavior of ants includes environmental exploration, discovery of route, setting and use of highly efficient routing paths.

Ant Colony Optimization [14] (ACO) is the most utilized optimization technique in swarm intelligence for approximation determination. ACO belongs to class of metaheuristics which are regarded as Approximation Algorithms and lay foundations for obtaining highly efficient solutions to $\mathrm{CO}$ problems in timely manner.

ACO, algorithms considering the optimization and efficiency has become an important source of foundation for researchers to develop algorithms for routing protocols for Wireless Sensor Networks. ACO, apart from WSN has also been applied to various other engineering disciplines and other areas of computer science for solving complex problems and determining optimized solutions.

\section{A. Ant Colony Optimization- Computational SI Technique- General Working and Algorithm}

Ant Colony Optimization (ACO),[14-22] was discovered by $\mathrm{M}$. Dorigo and colleagues for finding solutions to varied Hard CO problems in early 1990s. The basic foundation of ACO algorithms are real ant colonies. Ants roam randomly in the environment to determine food source and find the shortest path between food source and nest. In order to exchange information regarding which path to follow, ants communicate via use of chemical substance called Pheromone. As ants move from nest to food source, lay a trail of pheromone and other ants follow the same trail, laying trail of pheromone. The trail becomes more attractive when followed by huge majority of ants. Using this mechanism, ants are able to transport the food from source to nest in an efficient way.

\section{Ant Colony Optimization Algorithm}

$$
\begin{aligned}
& \text { input: An instance } P \text { of a } \mathrm{CO} \text { problem model } \mathcal{P}=(\mathcal{S}, f, \Omega) \text {. } \\
& \text { InitializePheromoneValues }(\mathcal{T}) \\
& \text { sbs } \leftarrow \text { NULL } \\
& \text { while termination conditions not met do } \\
& \Im_{\text {iter }} \leftarrow \emptyset \\
& \text { for } j=1, \ldots, n_{a} \text { do } \\
& \mathfrak{s} \leftarrow \text { ConstructSolution }(\mathcal{T}) \\
& \text { if } \mathfrak{s} \text { is a valid solution then } \\
& \mathfrak{s} \leftarrow \text { LocalSearch }(\mathfrak{s}) \quad \text { \{optional } \\
& \text { if }\left(f(\mathfrak{s})<f\left(\mathfrak{s}_{\mathrm{bs}}\right)\right) \text { or }\left(\mathfrak{s}_{\mathrm{bs}}=\text { NULL }\right) \text { then } \mathfrak{s}_{\mathrm{bs}} \leftarrow \mathfrak{s} \\
& \widetilde{\Xi}_{\text {iter }} \leftarrow \boldsymbol{\Xi}_{\text {iter }} \cup\{\mathfrak{s}\} \\
& \text { end if } \\
& \text { end for } \\
& \text { ApplyPheromoneUpdate }\left(\mathcal{T}, \Im_{\text {iter }}, \mathfrak{s} \text { bs }\right) \\
& \text { end while } \\
& \text { output: The best-so-far solution } \mathfrak{s}_{\mathrm{bs}}
\end{aligned}
$$

\section{B. ACO and Wireless Sensor Networks}

Wireless Sensor Networks (WSN), comprising thousands of autonomous and limited energy sensor nodes are deployed in wide range of environments. Efficient Routing is still demanding area to be researched out for developing efficient routing protocols for WSN.

Ant Colony Optimization based Algorithms comprise of varied distinguished features as listed below which makes it the most suitable for developing routing algorithms for Wireless Sensor Networks [23]:

1) As ACO algorithms are fully distributed, so failure rates are reduced to large extent in sensor nodes communications.

2) Simple operations can be performed in each and every node for routing of packets among nodes and back to sink node.

3) Autonomous integration of ants, and the algorithms are based on Agent's Synchronous.

4) ACO algorithms have capability of Self-Organizing which is very important as sensor nodes, when deployed randomly have to fully robust, scalable and fault tolerant. ACO algorithms makes WSN networks fully self-organization compliant.

5) ACO algorithms are very well suited to adapt to all kinds of changes in real-world topology and increase in number of nodes and packet traffic.

6) ACO algorithms solve complex $\mathrm{CO}$ problems, making them well suitable for highly complex situations when sensor 
nodes are deployed especially in Real-Time Monitoring, Production and Military based Battlefield's monitoring.

\section{REVIEW OF ROUTING PROTOCOLS FOR WSN BASED ON ANT Colony OptimizATION [23-30, 38, 40]}

In this section, Ant Colony Optimization based Routing Protocols for WSNs are highlighted.

\section{A. Sensor Driven Cost-Aware Ant Routing(SC) [31]}

The main problem surrounding all Basic Ant Routing Algorithms is that all the forwarding ants normally consume lots of time to locate the destination, even when a tabu list is being utilized (i.e. Repeating nodes are not included). This situation usually occurs when ants primarily don't have any idea regarding the exact destination. Only when the destination is located, the links are traversed along with certain probabilities of link exchange.

In SC Routing, the routing performance is improvised; it is assumed that forward ants are equipped with sensors to locate the best destination for food at the initial process of routing. In addition to smart sensing ability of ants, each node stores the probability distribution and every node estimates and stores the cost to the destination from neighboring nodes. It suffers from redundant data when obstacle arises in path leading to sensing errors.

SC Algorithm:
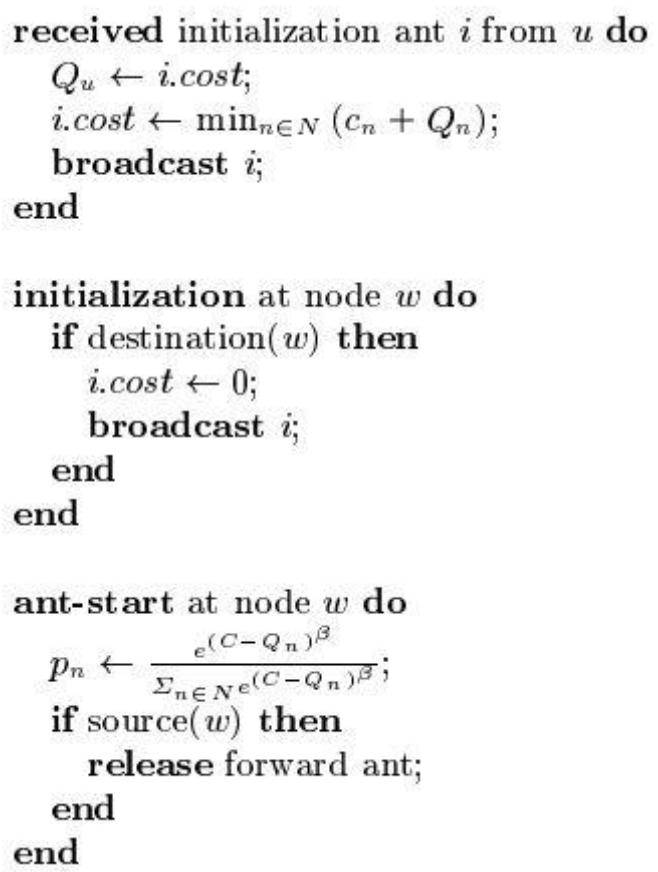

\section{B. Energy Efficient Ant Based Routing (EEABR) [32]}

Energy Efficient Ant Based Routing (EEABR) algorithm, proposed by T. Camilo et al [32] is an improvised routing protocol based on Ant Colony Optimization (ACO) metaheuristic. The protocol was designed with an objective to enhance sensor nodes energy by reducing communication overhead in discovering the paths from source to destination.
The protocol adds new functionalities in pheromone tables updation of sensor nodes.

\section{Algorithm}

1) In EEABR routing protocol, at regular interval period of time, from each network node, a forward ant is launched to determine a path from nest to food source. The identifier of every visited node is saved in memory and carried forward by ant. Each network node has routing table with $\mathrm{N}$ entries, one for each possible solution, and destination is one of the entry in nodes routing table.

2) At every node, the ant selects the next hop using the same ACO metaheuristic probabilistic rule.

3) When the forward ant reaches the food destination, it is transmitted back to proceeding ant, whose main task is to update the pheromone trail of the path used by forward ant to reach from nest to source and also stored in memory.

4) The destination node computes the amount of pheromone trail that the ant will drop during the journey, before backward ant starts the journey.

5) When the node, receives the backward ant coming from neighboring node, it updates the routing table.

6) When the backward ant reaches the nest, the actual path is determined by other ants to follow.

Simulation of EEABR with BABR (Basic Ant Based routing algorithm) and IABR (Improvised Ant-Based Routing Algorithm) is done on NS-2 simulator on varied parameters like Average Energy, Minimum Energy, Standard Deviation and Energy Efficiency and overall EEABR performs much better as compared to other two routing protocols. The only drawback of EEABR is lack of QoS and somewhat delay in packet delivery.

\section{Flooded Forward Ant Routing (FF) [31]}

Flooded Forward Ant Routing (FF) was developed to overcome the shortcomings of misguiding paths due to obstacles in SC protocol even when ants are equipped with sensors. When the exact destination is unknown at the beginning by ant and even the cost cannot be determined, SC protocol was reduced to Basic Ant Routing and still the problem of unknown wandering around the network by ant to find the destination exist. In that case, FF protocol was introduced to remove the problem.

FF protocol exploits the network via broadcast channel of WSN which means FF protocol makes use of Broadcast method of sensor networks to route the network packets from source to destination. The objective is to flood forward ants to the destination. If the food search is successful, forward ants will direct backward ants to traverse backwards to the source. Multiple paths are updated by one flooding phase and probabilities are updated in the same manner as in Basic Ant Routing Protocol.

\section{Flooded Piggyback Ant Routing (FP) [31]}

In flooded Piggyback Ant Routing (FP), a novel specimen of ants i.e. Data Ants was introduced. The forward list is carried by FP. In FP protocol, forward ants and data ants are 
combined via constrained flooding to route data packets and search for energy efficient paths in the network.

FP protocol was compared with $\mathrm{SC}$, FF and Basic ACO routing protocols in RMASE (Routing Modeling Application Simulation Environment) simulator. Results showed FP is not an energy efficient routing protocol. FF protocol is efficient in reducing delay and SC remains highly energy efficient routing protocol among FF, FP and Basic ACO routing protocol.

\section{E. Energy-Delay Ant Based (E-D Ants) [33]}

Energy-Delay Ant Based (E-D Ants) was proposed by Wen et. al (2008). E\&D ants is a reactive routing protocol being based on ant algorithms for performing varied routing operations. E-D Ants Protocol is based on Energy*Delay metrics to enhance network lifetime and minimize propagation delay by making use of a novel variation of Reinforcement Learning (RL).

The Mathematical expression of E-D Ants Protocol is:

$$
\mathrm{g}(\mathrm{t})=\min (\text { Energy } * \text { Delay })
$$

The protocol works on Iterative generation and unicast transmission of multiple forward ants to minimize energy and delay like AntNet Protocol. In this protocol, every ant stores the residual energy level and hop delay experience in its stack moving from node to node.

E-D Ants Routing protocol was simulated in OPNET Simulator using 50 sensor nodes in area of 100x100 m and compared with two routing protocols: AntNet and AntChain on basis of Energy Efficiency, Delay and Routing Overhead. The results showed E-D Ants Protocol is almost $150 \%$ efficient as compared to other two protocols. E-D Ants protocol is also efficient routing protocol in determining optimal paths from source to destination.

\section{F. Ant Colony Based Reinforcement Learning Algorithm (AR and IAR) [34-35]}

Adaptive Routing (AR) and Improved Adaptive Routing (IAR), proposed by Ghasemaghaei et. al (2007) uses probability distribution like other Ant-Colony based routing protocols in finding optimal paths from source to destination. The only difference between AR and IAR with other ACO based routing protocols is the use of reinforcement learning algorithm by backward ants to get efficient routing path from source to destination.

In AR and IAR, two types of ants are deployed:

1) Forward ant $\left(\mathrm{F}_{\mathrm{ant}}\right)$ - travelling from source node ( $\left.\mathrm{s}\right)$ to destination node (d)

2) Backward ant $\left(B_{\text {ant }}\right)$ : which is generated by $F_{a n t}$ when $F_{\text {ant }}$ reaches the destination $d$.

The backward ant gets back to sink node via information supplied by forward ant. But backward ant makes use of reinforcement learning method to get better and most optimal route as compared to the route being chosen by forward ant and updates the routing table of sensor nodes visited during reverse journey.

AR and IAR algorithms were simulated on Java based simulator using 7x7 sensor node grid for 200 seconds. AR and
IAR algorithms are compared with 4 Routing Algorithms: Basic Ant Routing, SC Ant Routing, FF and FP Routing Algorithm on parameters like Latency, Energy Consumption, Success Rates. Simulation results showed AR and IAR much efficient in every parameter as compared to other 4 routing protocols.

\section{G. Basic Ant Based Routing(BABR) for Wireless Sensor} Networks (WSN) [17] [21]

Ant Colony Optimization (ACO), is nature-inspired metaheuristic for solving complex Combinatorial Problems (CO). The main component of ACO algorithm is Pheromone Model.

ACO, being an optimization approach, used to solve complex problems by iterating the following two steps:

1) Using a Pheromone model, that is, a parametrized probability distribution over the solution space;

2) The candidate solutions are used to modify the pheromone values in a way that is deemed to bias future sampling forward high quality solutions.

Basic Ant Based Routing leads to the development of AntNet Algorithm which can be summarized as follows:

a) Forward ant is launched from source node to sink node to determine the optimal path to destination.

$b$ ) The main task of forward ant is to locate the food source with equal probability by using neighboring nodes with minimum cost joining its source to sink.

c) As ants move forward from node to node to reach the destination, the routing table gets updated side by side.

d) Forward ants calculate all the information about the time length, congestion status and the node identifiers of the followed path.

e) On reaching the destination node, the backward ant is created which follows the same path as forward ant, in opposite direction i.e. from food source to nest.

f) During backward travel, local models of the network status and the local routing table of each visited node are modified by the agents as a function of the path they followed and of its goodness.

\section{H. Ant Based Quality of Service Routing (ACO-QoSR) [36]}

ACO-QoSR, a reactive routing algorithm was developed by Cai et. al in 2006 to tackle problems of constraint delay and energy in Wireless Sensor Networks. The basic objective behind the development of ACO-QoSR routing is to find optimal routes between varied sensor nodes to sink node in such a way that the total end-to-end delay is less than a boundary value, while the energy residual ratio i.e. $\mathrm{ERR}=\mathrm{E}_{\text {residual }} / \mathrm{E}_{\text {initial }}$ is above a certain value.

\section{ACO-QoSR Algorithm}

When source node wants to send data, it first checks routing table to determine optimal path. Route probing will only start if there are no unexpired paths to the destination, and node needs to cache data waiting for transmission at the same time. Forwards ants does the task for route probing and after route discovery cached data is sent to destination in no 
time. In order to reduce time delay of route discovery, ACOQoSR algorithm starts a full route probe phase at the time of network initialization.

Forward Ant Phase: In forward ant's phase, if the sending/source sensor unable to find a favorable path to sink node in routing table, it will generate a number of forward ants to search for optimal paths to destination. Forward ants will establish pheromone track between source to destination node. Forward ants comprise of various parameters: Timestamp origin, source and destination address. The main aim of forward ant is to collect intermediate node's local information and record the path information of various nodes from source to destination.

Backward Ants Phase: When the forward ant reaches the destination, the forward ant will be killed and backward ant will be generated which carries source and destination address, backward ant ID, path information from forward ant and pheromone update value.

Route Maintenance Phase: The entries in the routing table are basically pheromone values and probabilities that next-hop is a specific neighbor. Probabilities allow the ants to roam randomly in the environment and find new and optimal paths. Once the new optimal paths are discovered, the next hop probabilities are updated to routing table to reflect new paths from source nodes to sink nodes.

ACO-QoSR protocol was simulated in NS-2 Simulator [38] considering the network of 100 sensor nodes in 1000x1000m area. ACO-QoSR protocol was compared with AODV and DSDV protocols on parameters like end-to-end delay, packet delivery ratio, routing overhead and path's normalized energy residual ratio. Simulation results showed that ACO-QoSR has better energy residual ratio and less overhead but packet delivery ratio is just average as compared to AODV and DSDV and routing overhead is small.

\section{Ant Colony Optimization based Location-aware Routing (ACLR) [37]}

Ant Colony Optimization based Location Aware Routing (ACLR), a High Performance Routing Protocol for Wireless Sensor Networks was designed by Wang et. al in 2008. The principle behind the working of ACLR protocol is determination and selection of next hop by ants to a subset of the set of the neighbors of the current node which guarantee for the packet delivery rather than searching of whole neighbors to avoid loops. The protocol also determines the amount of pheromone which laid by the ant from source node to sink node. In addition, the protocol also proposes a novel scheme to evaporate the pheromone on the different segments of a certain route as per residual energy and the location information of nodes.

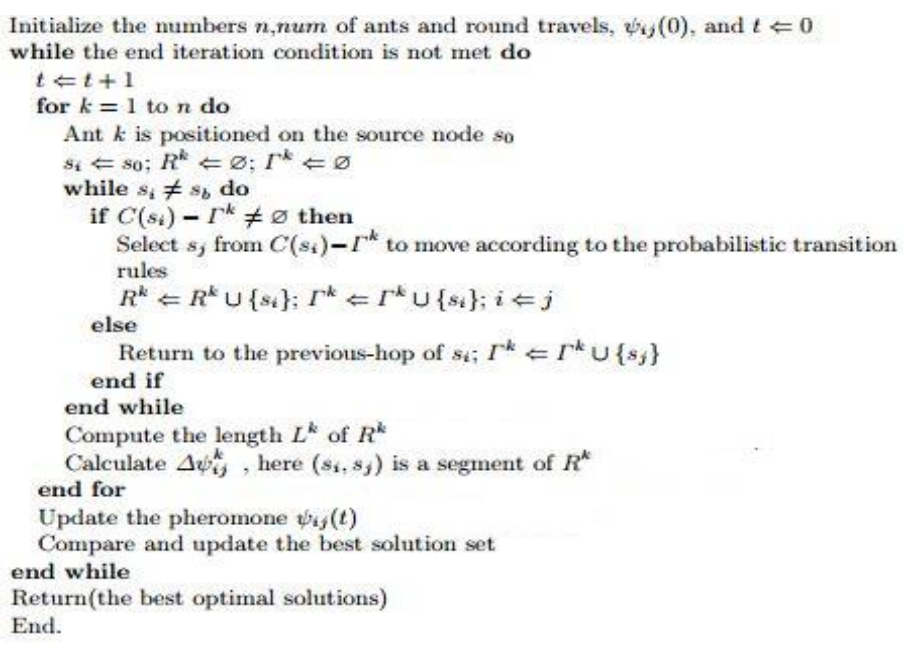

ACLR Algorithm

ACLR Algorithm was simulated on OPNET Simulator and compared with 4 algorithms: Basic Ant Routing (BAR), SC, FP and IAR using network area of $200 \times 300 \mathrm{~m}$ and 10000 sensors. Performance of ACLR algorithm is determined on energy consumption, efficiency and packet delivery latency. Results showed that ACLR consumes less energy as compared to 4 other algorithms and it is also better in terms of Packet Delivery.

\section{CONCLUSION AND FUTURE DIRECTIONS}

Wireless Sensor Networks, being strongest platform for research across wide forum of researchers around the world. Wireless Sensor Network nodes are resource-constrained nodes and lots have to be done regarding improvement of various parameters to make WSN network more adaptable in real world.

The design and development of energy efficient, robust, scalable and effective packet delivery routing protocol in WSN network is a challenging task. Diverse optimization fields like Swarm Intelligence, Fuzzy Logics, Genetic Algorithms are being utilized by researchers to develop routing protocols. One of the most utilized novel domain in development in WSN routing protocols is Swarm Intelligence. So, taking SI into consideration wide range of protocols are developed and still lots are under rapid development and testing phase by researchers, most specifically, taking two main techniques into consideration: Ant Colonies: Based on foraging behavior of Ants and Bee Colonies: Considering efficient way of communication of Bees.

In this paper, we have presented a detailed comprehensive review of Ant Colony Optimization based Routing Protocols for Wireless Sensor Networks. 
Considering all the protocols in this paper, it is being observed that currently very little research is being done regarding Security, QoS parameters and most of the protocols assume that sink node is stationary which is a limited barrier towards research. Research should be done seriously considering each and every node in topology to be dynamic and mobile and having random changing scenario. New routing Protocols which are required in WSN should be able to handle mobility overhead and random topology changes by maintaining optimal paths in route discovery, selection and maintenance and especially strong consideration should be done in maintaining energy efficiency of each and every node in the network.

On other hand, apart from developing and testing the new routing protocols over Simulators and Testbeds, it is also recommended that research should be conducted on live sensor nodes for determining accurate performance on the basis of algorithm/protocol proposed.

We strongly believe that considering this paper, researchers would take SI into more serious consideration and come up with more advanced and efficient routing protocols well tested with diverse parameters and fully functional to be adaptable in real world sensor networks.

\section{FUTURE SCOPE}

In near future, considering the pros and cons of different routing protocols being developed for WSN using Ant Colony Optimization, a Novel Multipath based routing protocol more efficient in packet delivery, end-to-end delay, less routing overhead and Energy Efficiency will be developed.

\section{REFERENCES}

[1] Akkaya, K., \& Younis, M. (2005). A survey on routing protocols for wireless sensor networks. Ad hoc networks, 3(3), 325-349.

[2] Akyildiz, I. F., Su, W., Sankarasubramaniam, Y., \& Cayirci, E. (2002). Wireless sensor networks: a survey. Computer networks, 38(4), 393-422.

[3] Chong, C. Y., \& Kumar, S. P. (2003). Sensor networks: evolution, opportunities, and challenges. Proceedings of the IEEE, 91(8), 12471256.

[4] Kephart, J. O., \& Chess, D. M. (2003). The vision of autonomic computing. Computer, 36(1), 41-50.

[5] Bonabeau, E., Dorigo, M., \& Theraulaz, G. (1999). Swarm intelligence: from natural to artificial systems (No. 1). Oxford university press.

[6] Kennedy, J., Kennedy, J. F., Eberhart, R. C., \& Shi, Y. (2001). Swarm intelligence. Morgan Kaufmann.

[7] Engelbrecht, A. P. (2006). Fundamentals of computational swarm intelligence. John Wiley \& Sons.

[8] Keerthi, S., Ashwini, K., \& Vijaykumar, M. V. (2015). Survey Paper on Swarm Intelligence. International Journal of Computer Applications, 115(5).

[9] Di Caro, G. A. (2014). Principles of swarm intelligence for adaptive routing telecommunication networks. Sistemi intelligenti, 26(3), 443464.

[10] Raghavendra, C. S., Sivalingam, K. M., \& Znati, T. (Eds.). (2006). Wireless sensor networks. Springer.

[11] Zheng, J., \& Jamalipour, A. (2009). Wireless sensor networks: a networking perspective. John Wiley \& Sons.

[12] Misra, S., Zhang, I., \& Misra, S. C. (Eds.). (2009). Guide to wireless sensor networks. Springer Science \& Business Media.

[13] Goyal, D., \& Tripathy, M. R. (2012, January). Routing protocols in wireless sensor networks: a survey. In 2012 Second International
Conference on Advanced Computing \& Communication Technologies (pp. 474-480). IEEE.

[14] Dorigo, M., Birattari, M., \& Stutzle, T. (2006). Ant colony optimization. IEEE computational intelligence magazine, 1(4), 28-39.

[15] Yaseen, S. G., \& Al-Slamy, N. M. (2008). Ant colony optimization. IJCSNS, 8(6), 351.

[16] Stützle, T. (2009, April). Ant colony optimization. In International Conference on Evolutionary Multi-Criterion Optimization (pp. 2-2). Springer Berlin Heidelberg.

[17] Dorigo, M., \& Blum, C. (2005). Ant colony optimization theory: A survey. Theoretical computer science, 344(2), 243-278.

[18] Dorigo, M., \& Stützle, T. (2003). The ant colony optimization metaheuristic: Algorithms, applications, and advances. In Handbook of metaheuristics (pp. 250-285). Springer US.

[19] Blum, C. (2005). Ant colony optimization: Introduction and recent trends. Physics of Life reviews, 2(4), 353-373.

[20] Sim, K. M., \& Sun, W. H. (2003). Ant colony optimization for routing and load-balancing: survey and new directions. IEEE Transactions on Systems, Man, and Cybernetics-Part A: Systems and Humans, 33(5), 560-572.

[21] Nayyar, A., \& Singh, R. (2016, October). Ant Colony OptimizationComputational swarm intelligence technique. In Computing for Sustainable Global Development (INDIACom), 2016 3rd International Conference on (pp. 1493-1499). IEEE.

[22] Maniezzo, V., \& Carbonaro, A. (2002). Ant colony optimization: an overview. In Essays and surveys in metaheuristics (pp. 469-492). Springer US.

[23] Farooq, M., \& Di Caro, G. A. (2008). Routing protocols for nextgeneration networks inspired by collective behaviors of insect societies: An overview. In Swarm Intelligence (pp. 101-160). Springer Berlin Heidelberg.

[24] Saleem, M., Di Caro, G. A., \& Farooq, M. (2011). Swarm intelligence based routing protocol for wireless sensor networks: Survey and future directions. Information Sciences, 181(20), 4597-4624.

[25] Zungeru, A. M., Ang, L. M., \& Seng, K. P. (2012). Classical and swarm intelligence based routing protocols for wireless sensor networks: A survey and comparison. Journal of Network and Computer Applications, 35(5), 1508-1536.

[26] Zengin, A., \& Tuncel, S. (2010). A survey on swarm intelligence based routing protocols in wireless sensor networks. International Journal of Physical Sciences, 5(14), 2118-2126.

[27] Ali, Z., \& Shahzad, W. (2011, July). Critical analysis of swarm intelligence based routing protocols in adhoc and sensor wireless networks. In Computer Networks and Information Technology (ICCNIT), 2011 International Conference on (pp. 287-292). IEEE.

[28] Wang, C., \& Lin, Q. (2008, June). Swarm intelligence optimization based routing algorithm for Wireless Sensor Networks. In Neural Networks and Signal Processing, 2008 International Conference on (pp. 136-141). IEEE.

[29] Geetha, R., \& Srikanth, G. U. (2012). Ant Colony optimization based Routing in various Networking Domains-A Survey. International Research Journal of Mobile and Wireless Communications, 3(01), 424428.

[30] Nayyar, A., \& Singh, R. (2016). A Comprehensive Review of Ant Colony Optimization (ACO) Based Energy-Efficient Routing Protocols for Wireless Sensor Networks.

[31] Zhang Y, Kuhn LD, Fromherz MPJ (2004). "Improvements on Ant Routing for SensorNetworks," Ant Colony, Optimization And Swarm Intelligence, Lecture Notes in Computer Science, 2004, 3172: 289-313.

[32] Camilo, T., Carreto, C., Silva, J. S., \& Boavida, F. (2006, September). An energy-efficient ant-based routing algorithm for wireless sensor networks. In International Workshop on Ant Colony Optimization and Swarm Intelligence (pp. 49-59). Springer Berlin Heidelberg.

[33] Wen, Y. F., Chen, Y. Q., \& Pan, M. (2008). Adaptive ant-based routing in wireless sensor networks using Energy* Delay metrics. Journal of Zhejiang University SCIENCE A, 9(4), 531-538.

[34] GhasemAghaei, R., Rahman, A. M., Rahman, M. A., Gueaieb, W., \& El Saddik, A. (2008, March). Ant colony-based many-to-one sensory data 
routing in wireless sensor networks. In 2008 IEEE/ACS International Conference on Computer Systems and Applications (pp. 1005-1010). IEEE.

[35] GhasemAghaei, R., Rahman, M. A., Gueaieb, W., \& El Saddik, A. (2007, May). Ant colony-based reinforcement learning algorithm for routing in wireless sensor networks. In 2007 IEEE Instrumentation \& Measurement Technology Conference IMTC 2007 (pp. 1-6). IEEE.

[36] Cai, W., Jin, X., Zhang, Y., Chen, K., \& Wang, R. (2006, September). ACO based QoS routing algorithm for wireless sensor networks. In International Conference on Ubiquitous Intelligence and Computing (pp. 419-428). Springer Berlin Heidelberg.
[37] Wang, X., Li, Q., Xiong, N., \& Pan, Y. (2008, October). Ant colony optimization-based location-aware routing for wireless sensor networks. In International Conference on Wireless Algorithms, Systems, and Applications (pp. 109-120). Springer Berlin Heidelberg.

[38] Nayyar, A., \& Singh, R. (2015). A Comprehensive Review of Simulation Tools for Wireless Sensor Networks (WSNs). Journal of Wireless Networking and Communications, 5(1), 19-47.

[39] Nayyar, A., \& Sharma, S. (2014). A Survey on Coverage and Connectivity Issues Surrounding Wireless Sensor Network. IJRCCT, 3(1), 111-118.

[40] Kumar, A., \& Nayyar, A. Energy Efficient Routing Protocols for Wireless Sensor Networks (WSNs) based on Clustering. 